\title{
Prognostic value of tripartite motif containing 29 expression in patients with gastric cancer following surgical resection
}

\author{
CHENGHU WANG $^{1}$, YI ZHOU ${ }^{2}$, BEIBEI CHEN ${ }^{3}$, WEIWEI YUAN ${ }^{1}$ and JINXI HUANG ${ }^{1}$ \\ Departments of ${ }^{1}$ General Surgery, ${ }^{2}$ Anesthesiology and ${ }^{3}$ Internal Medicine of Digestive Tract Tumor, \\ The Affiliated Cancer Hospital of Zhengzhou University, Zhengzhou, Henan 450003, P.R. China
}

Received April 13, 2016; Accepted September 15, 2017

DOI: $10.3892 / \mathrm{ol} .2018 .8059$

\begin{abstract}
Tripartite motif containing 29 (TRIM29) dysregulation serves an important function in the progression of numerous types of cancer, but its function in the prognosis of patients with gastric cancer remains unknown. The present study assessed the prognostic value of TRIM29 in patients with gastric cancer following surgical resection. A total of 243 fresh gastric adenocarcinoma and adjacent normal tissues were continuously retrieved from patients who underwent curative surgery for gastric cancer at the Cancer Hospital of Henan Province (Zhengzhou, China) between January 2005 and December 2011. The reverse transcription-quantitative polymerase chain reaction was performed to assess TRIM29 expression. The association between TRIM29 expression and clinicopathological features and prognosis was subsequently evaluated. The results of the present study revealed that the expression of TRIM29 was increased in the gastric cancer tissues compared with the normal adjacent tissues, and that upregulated expression of TRIM29 was associated with tumor cell differentiation, tumor stage, lymph node metastasis, and tumor-node-metastasis (TNM) stage. In the training and validation data, high TRIM29 expression was associated with poor overall survival in patients with gastric cancer. Furthermore, multivariate analysis identified that TRIM29 expression was an independent prognostic factor for overall survival, in addition to TNM stage and Lauren classification. Combining TRIM29 expression with the TNM staging system generated a novel predictive model that exhibited improved prognostic accuracy for overall survival in patients with gastric cancer. The present study revealed that TRIM29 was an independent adverse prognostic factor in patients with gastric cancer. Incorporating TRIM29 expression level into the TNM staging system may improve risk stratification
\end{abstract}

Correspondence to: Dr Jinxi Huang, Department of General Surgery, The Affiliated Cancer Hospital of Zhengzhou University, 127 Dongming Road, Zhengzhou, Henan 450003, P.R. China E-mail: jinxihuang2016@sohu.com

Key words: tripartite motif containing 29, gastric cancer, prognostic value, radical gastrectomy, overall survival and render prognosis more accurate in patients with gastric cancer.

\section{Introduction}

Despite advances in surgery, chemoradiotherapy, and molecular-targeted therapy, the prognosis for patients with advanced gastric cancer tends to be poor (1-3). The tumor-node-metastasis (TNM) staging system of the American Joint Committee on Cancer is a useful model for predicting the prognosis of patients with gastric cancer $(4,5)$. However, patients with gastric cancer with the same TNM stage may receive different prognoses, partly due to heterogeneity at the molecular level of the disease; an example of this is the different prognosis of patients with gastric cancer with the same TNM stage in East Asia and Europe (6,7). The identification of specific and sensitive markers to supplement the TNM stage is required to treat patients with gastric cancer more precisely.

Tripartite motif containing 29 (TRIM29), located at chromosome 11q23, was initially identified in a study researching the gene responsible for the genetic disorder ataxia telangiectasia (8). TRIM29 belongs to the TRIM protein family, which is characterized by a Really Interesting New Gene (RING) finger domain, a B-box-type zinc finger domain type (B)1, a B2, and a coiled coil region (CC) (9). Unlike the majority of TRIM proteins, TRIM29 possesses B1-B2-CC domains but lacks the RING finger domain, suggesting that TRIM29 exhibits no E3 ubiquitin ligase activity (9). TRIM29 has been demonstrated to control important cellular processes, including intracellular signaling in innate immunity and viral infection, transcriptional regulation, development, autophagy and carcinogenesis (10). Previous pathological studies have revealed that TRIM29 may be useful in the diagnosis of multiple types of cancer, including breast (11), prostate (12), pancreatic (13), lung (14) and bladder cancer (15), and esophageal squamous cell carcinoma (16). The expression and biological functions of TRIM29 differ between different types of cancer (10). A previous study demonstrated that TRIM29 expression was a marker for lymph node metastasis in gastric cancer (17). However, the function of TRIM29 in the prognosis of patients with gastric cancer remains to be fully understood and requires further study. 
The present study assessed the potential function of TRIM29 expression in the prognosis of patients with resectable gastric cancer. TRIM29 expression was evaluated using the reverse transcription-quantitative polymerase chain reaction (RT-qPCR) in gastric cancer tissues. The association between TRIM29 expression and clinical outcomes in patients with gastric cancer was assessed. The results of the present study may assist in evaluating the clinical significance of TRIM29 expression in gastric cancer and provide means for a more precise prognostic system for evaluating outcomes in patients with gastric cancer.

\section{Materials and methods}

Clinical specimens. A total of 243 fresh gastric adenocarcinoma and adjacent normal tissues were continuously retrieved from patients who underwent curative surgery (R0 resection and D2 lymphadenectomy) for gastric cancer at the Cancer Hospital of Henan Province (Zhengzhou, China) between January 2005 and December 2011. All surgeries were performed according to the guidelines of the International Gastric Cancer Association and the Japanese Gastric Cancer Association (5). The patients enrolled in the present study provided written informed consent for publication and were divided into two independent sets (all clinicopathological data for the patients is provided in Table I). Tissues from the training set $(n=113)$ were collected between January 2005 and December 2008. Tissues from the validation set $(n=130)$ were collected between January 2009 and December 2011. The fresh cancer tissues were immediately frozen $\left(-180^{\circ} \mathrm{C}\right)$ and stored in liquid nitrogen following resection, until further analysis. Pathological examinations were performed and the histological evaluation was reassessed independently by two gastroenterology pathologists of the Cancer Hospital of Henan Province according to the Japanese General Rules for Gastric Cancer Study in Surgery and Pathology (5), and the TNM staging system was applied according to the 2010 International Union Against Cancer TNM classification system (4). Patients were excluded if they had previously been exposed to any radiotherapy, chemotherapy, targeted therapy, or intervention therapy for gastric cancer. The present study was reviewed and approved by the Clinical Research Ethics Committee of the Cancer Hospital of Henan Province.

$R T$-qPCR. TRIM29 expression was evaluated using RT-qPCR, which was performed as previously described (11). Briefly, total RNA containing microRNA (miRNA) was extracted from cultured cells or tissues using the miRNeasy Mini kit (Qiagen, Inc., Valencia, CA, USA), according to manufacturer's protocol. cDNA was synthesized using the miScript Reverse Transcription kit (Qiagen, Inc.) following the manufacturer's protocol. Reverse transcription was performed using $50 \mathrm{ng}$ total RNA with a primer specific for TRIM29, together with the SYBR Green microRNA reverse transcription kit (Applied BioSystems; Thermo Fisher Scientific, Inc., Waltham, MA, USA), according to manufacturer's protocol. miRNAs were quantified using the SYBR Green miRNA RT-qPCR assay (Applied BioSystems; Thermo Fisher Scientific, Inc.) according to the manufacturer's protocol. The qPCR reaction was carried out on a 7500 Fast Real-time System (Applied Biosystems; Thermo Fisher Scientific, Inc.). All RT-qPCRs were performed in triplicate. The data were analyzed using an automated baseline. The threshold cycle $(\mathrm{Cq})$ was defined as the fractional cycle number at which the fluorescence exceeded the given threshold. The data obtained from the RT-qPCR were analyzed using the $\Delta \Delta \mathrm{Cq}$ method $\left(2^{\Delta \Delta \mathrm{Cq}}\right)(11)$. The primer sequences used for RT-qPCR were as follows: TRIM29 forward, 5'-ACATCATACCAGCCCTCGTC-3' and reverse, 5'-AGCCTTTCAGGGAGAAGGAG-3'; RNA, U6 small nuclear 1 (U6) forward, 5'-GCTTCGGCAGCACAT ATACTAAAAT-3' and reverse, 5'-CGCTTCACGAATTTG CGTGTCAT-3'. U6 was used as the internal control.

Statistical analysis. Statistical analysis was performed using SPSS Statistics 21.0 software (IBM Corp., Armonk, NY, USA). Pearson's $\chi^{2}$ tests or Fisher's exact tests were applied for categorical variables. Survival curves were constructed using the Kaplan-Meier method and the significance of the difference between survival curves was assessed using the log-rank test. Numbers at risk were calculated for the beginning of each time period. The Cox proportional hazards regression model was used for multivariate analysis. Receiver operating characteristic (ROC) analysis was used to compare the sensitivity and specificity for the prediction of overall survival by the parameters. All $\mathrm{P}$-values were two-sided. $\mathrm{P}<0.05$ was considered to indicate a statistically significant difference.

\section{Results}

Association between TRIM29 expression and pathological characteristics. To evaluate whether TRIM29 expression is associated with the development and progression of gastric cancer, the present study assessed TRIM29 expression in 243 patients with gastric cancer using RT-qPCR analysis. Using the results of RT-qPCR, patients were divided into high or low expression groups according to the ratio of the mean expression level of TRIM29 in their normal tissue to that in their cancer tissue (cut-off was 0.5 ). In the training and validation sets, $58.41(66 / 113)$ and $53.08 \%$ (69/130) of the tumors were determined to exhibit high TRIM29 expression, respectively. The characteristics and clinicopathological features of the patients were provided (Table I). TRIM29 expression was significantly associated with tumor cell differentiation (training set, $\mathrm{P}=0.001$; validation set, $\mathrm{P}=0.001$ ), tumor stage (training set, $\mathrm{P}<0.001$; validation set, $\mathrm{P}=0.016$ ), lymph node metastasis (training set, $\mathrm{P}=0.016$; validation set, $\mathrm{P}<0.001$ ), and TNM stage (training set, $\mathrm{P}=0.005$; validation set, $\mathrm{P}=0.001$ ). Furthermore, the percentage of patients with high TRIM29 expression increased with cancer progression from TNM stage I to III in the training and validation sets (Fig. 1).

Survival analysis to evaluate the prognostic value of TRIM29 in patients with resectable gastric cancer. In the training and validation sets, high TRIM29 expression was associated with poorer overall survival than those with low TRIM29 expression (training set, $\mathrm{P}<0.001$; validation set, $\mathrm{P}<0.001$; Fig. 2), which suggested that TRIM29 expression influenced the clinical outcome for patients with resectable gastric cancer. Furthermore, univariate analysis demonstrated that high TRIM29 expression is a significant negative prognostic predictor for patients with gastric cancer in the training [hazard 
Table I. Association between TRIM29 expression and clinical characteristics in patients with gastric cancer.

\begin{tabular}{|c|c|c|c|c|c|c|}
\hline \multirow[b]{3}{*}{ Factor } & \multicolumn{3}{|c|}{ Training set } & \multicolumn{3}{|c|}{ Validation set } \\
\hline & \multicolumn{3}{|c|}{ TRIM29 expression } & \multicolumn{3}{|c|}{ TRIM29 expression } \\
\hline & Low (n) & $\operatorname{High}(\mathrm{n})$ & P-value & Low (n) & $\operatorname{High}(\mathrm{n})$ & P-value \\
\hline All patients & 47 & 66 & & 61 & 69 & \\
\hline Age/years ${ }^{\mathrm{a}}$ & & & 0.272 & & & 0.245 \\
\hline$\leq 60$ & 20 & 35 & & 23 & 33 & \\
\hline$>60$ & 27 & 31 & & 38 & 36 & \\
\hline Sex & & & 0.104 & & & 0.292 \\
\hline Female & 36 & 41 & & 38 & 49 & \\
\hline Male & 11 & 25 & & 23 & 20 & \\
\hline Localization & & & 0.174 & & & 0.593 \\
\hline Proximal & 3 & 7 & & 10 & 12 & \\
\hline Middle & 23 & 21 & & 18 & 15 & \\
\hline Distal & 21 & 38 & & 33 & 42 & \\
\hline Differentiation & & & 0.001 & & & 0.001 \\
\hline Well & 8 & 1 & & 15 & 7 & \\
\hline Moderately & 25 & 25 & & 26 & 17 & \\
\hline Poorly & 14 & 40 & & 20 & 45 & \\
\hline Lauren classification & & & 0.459 & & & 0.202 \\
\hline Intestinal & 31 & 39 & & 25 & 36 & \\
\hline Diffuse & 16 & 27 & & 36 & 33 & \\
\hline Tumor stage & & & $<0.001$ & & & 0.016 \\
\hline $1+2$ & 25 & 13 & & 16 & 7 & \\
\hline $3+4$ & 22 & 53 & & 45 & 62 & \\
\hline Lymph node metastasis & & & 0.016 & & & $<0.001$ \\
\hline Negative & 19 & 13 & & 34 & 14 & \\
\hline Positive & 28 & 53 & & 27 & 55 & \\
\hline TNM stage & & & 0.005 & & & 0.001 \\
\hline I & 18 & 8 & & 14 & 6 & \\
\hline II & 9 & 17 & & 22 & 13 & \\
\hline III & 20 & 41 & & 35 & 50 & \\
\hline Tumor size $/ \mathrm{cm}^{\mathrm{a}}$ & & & 0.157 & & & 0.316 \\
\hline$<4.0$ & 23 & 27 & & 31 & 29 & \\
\hline$\geq 4.0$ & 24 & 39 & & 30 & 40 & \\
\hline
\end{tabular}

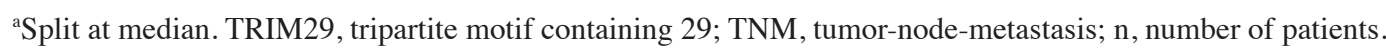

ratio (HR), 5.14; 95\% confidence interval (CI), 2.49-10.63; $\mathrm{P}<0.001]$ and validation sets $(\mathrm{HR}, 5.22 ; 95 \% \mathrm{CI}, 2.44-11.14$; $\mathrm{P}<0.001$ ). Furthermore, tumor cell differentiation (training set, $\mathrm{P}=0.001$; validation set, $\mathrm{P}=0.010$ ), Lauren classification (training set, $\mathrm{P}=0.002$; validation set, $\mathrm{P}=0.046$ ), tumor stage (training set, $\mathrm{P}<0.001$; validation set, $\mathrm{P}=0.007$ ), lymph node metastasis (training set, $\mathrm{P}=0.001$; validation set, $\mathrm{P}<0.001$ ), and TNM stage (training set, $\mathrm{P}<0.001$; validation set, $\mathrm{P}<0.001$ ) significantly affected the survival of patients with gastric cancer (Table II). In addition, using multivariate Cox regression analysis, Lauren classification, TNM stage, and TRIM29 expression were identified as independent and significant prognostic parameters in the training (Lauren classification:

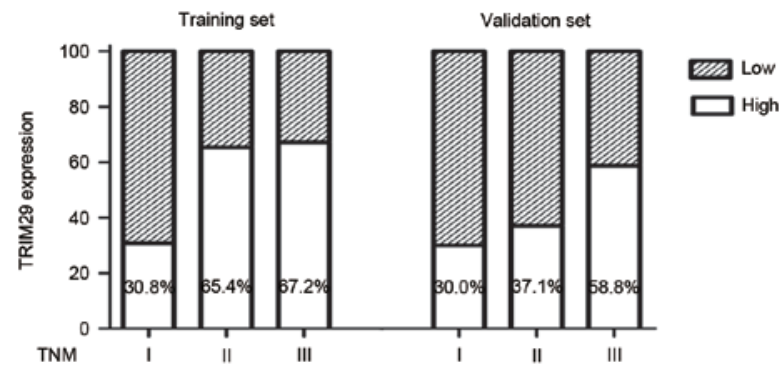

Figure 1. TRIM29 expression in patients with gastric cancer with different TNM stages. The percentage of patients with low TRIM29 expression increased with disease progression from TNM stage I to III in the training and validation sets. TRIM29, tripartite motif containing 29; TNM, tumor-node-metastasis. 
Table II. Univariate Cox regression analysis for overall survival in patients with gastric cancer.

\begin{tabular}{|c|c|c|c|c|}
\hline \multirow[b]{3}{*}{ Factor } & \multicolumn{4}{|c|}{ Overall survival } \\
\hline & \multicolumn{2}{|c|}{ Training set } & \multicolumn{2}{|c|}{ Validation set } \\
\hline & $\mathrm{HR}(95 \% \mathrm{CI})$ & P-value & $\mathrm{HR}(95 \% \mathrm{CI})$ & P-value \\
\hline Age/years ${ }^{\mathrm{a}}$ & & 0.201 & & 0.381 \\
\hline$\leq 60$ & 1.00 & & 1.00 & \\
\hline$>60$ & $1.45(0.82-2.54)$ & & $1.29(0.73-2.30)$ & \\
\hline Sex & & 0.357 & & 0.876 \\
\hline Female & 1.00 & & 1.00 & \\
\hline Male & $1.24(0.57-1.69)$ & & $0.95(0.53-1.73)$ & \\
\hline Localization & & 0.632 & & 0.515 \\
\hline Proximal + middle & 1.00 & & 1.00 & \\
\hline Distal & $1.15(0.66-2.00)$ & & $0.90(0.65-1.24)$ & \\
\hline Differentiation & & 0.001 & & 0.010 \\
\hline Well + moderately & 1.00 & & 1.00 & \\
\hline Poorly & $1.67(1.24-2.25)$ & & $1.47(1.10-1.96)$ & \\
\hline Lauren classification & & 0.002 & & 0.046 \\
\hline Intestinal & 1.00 & & 1.00 & \\
\hline Diffuse & $2.41(1.38-4.21)$ & & $1.69(1.21-3.02)$ & \\
\hline Tumor stage & & $<0.001$ & & 0.007 \\
\hline $1+2$ & 1.00 & & 1.00 & \\
\hline $3+4$ & $7.66(3.02-19.43)$ & & $15.25(2.10-110.62)$ & \\
\hline Lymph node metastasis & & 0.001 & & $<0.001$ \\
\hline Negative & 1.00 & & 1.00 & \\
\hline Positive & $4.04(1.72-9.51)$ & & $11.42(4.06-32.13)$ & \\
\hline TNM stage & & $<0.001$ & & $<0.001$ \\
\hline I+II & 1.00 & & 1.00 & \\
\hline III & $3.82(2.02-7.23)$ & & $12.30(4.80-31.55)$ & \\
\hline Tumor size, $\mathrm{cm}^{\mathrm{a}}$ & & 0.205 & & 0.174 \\
\hline$<4.0$ & 1.00 & & 1.00 & \\
\hline$\geq 4.0$ & $1.43(0.82-2.50)$ & & $1.56(0.72-3.50)$ & \\
\hline TRIM29 expression & & $<0.001$ & & $<0.001$ \\
\hline Low & 1.00 & & 1.00 & \\
\hline High & $5.14(2.49-10.63)$ & & $5.22(2.44-11.14)$ & \\
\hline
\end{tabular}

asplit at median. HR, hazard ratio; CI, confidence interval; TNM, tumor-node-metastasis; TRIM29, tripartite motif containing 29.
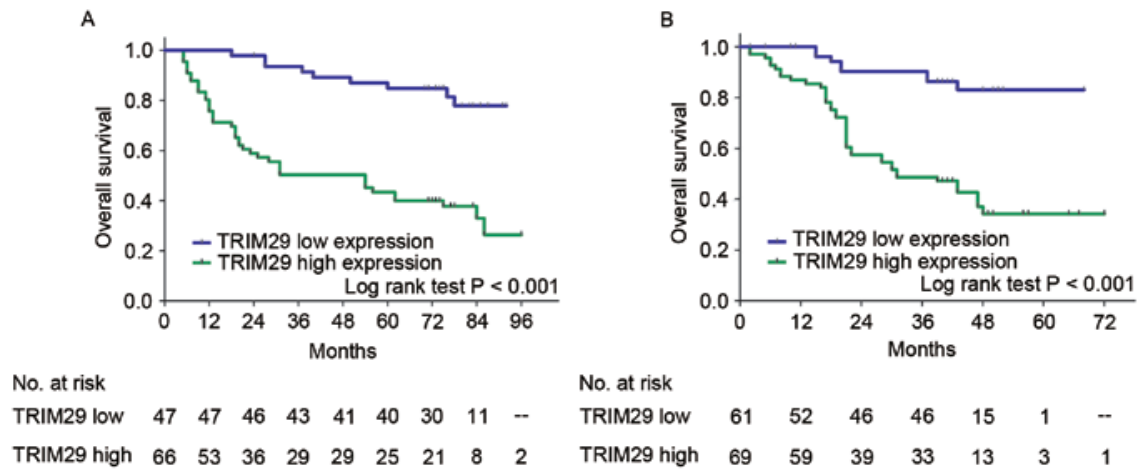

Figure 2. Analysis of overall survival according to TRIM29 expression in patients with gastric cancer. Kaplan-Meier analysis of overall survival according to TRIM29 expression in (A) the training $(\mathrm{n}=113)$ and $(\mathrm{B})$ validation sets $(\mathrm{n}=130)$. P-values were calculated using the log-rank test. TRIM29, tripartite motif containing $29 ; \mathrm{n}$, number of patients. 
Table III. Multivariate Cox regression analysis for overall survival in patients with gastric cancer.

A, Training set

\begin{tabular}{|c|c|c|c|c|}
\hline \multirow[b]{3}{*}{ Factor } & \multicolumn{4}{|c|}{ Overall survival } \\
\hline & \multicolumn{2}{|c|}{ Patients } & \multicolumn{2}{|c|}{ Multivariate analysis } \\
\hline & No. & $\%$ & $\operatorname{HR}(95 \% \mathrm{CI})$ & P-value \\
\hline All training set patients & 113 & 100 & & \\
\hline Differentiation & & & & 0.446 \\
\hline Well + moderately & 59 & 52.2 & 1.00 & \\
\hline Poorly & 54 & 47.8 & $1.17(0.61$ to 2.26$)$ & \\
\hline Lauren classification & & & & 0.001 \\
\hline Intestinal & 70 & 61.9 & 1.00 & \\
\hline Diffuse & 43 & 38.1 & 2.81 (1.56 to 5.09$)$ & \\
\hline TNM stage & & & & $<0.001$ \\
\hline I+II & 52 & 46.0 & 1.00 & \\
\hline III & 61 & 54.0 & $4.93(2.32$ to 10.49$)$ & \\
\hline TRIM29 expression & & & & $<0.001$ \\
\hline Low & 47 & 41.6 & 1.00 & \\
\hline High & 66 & 58.4 & $5.68(2.58$ to 12.51$)$ & \\
\hline
\end{tabular}

B, Validation set

\begin{tabular}{|c|c|c|c|c|}
\hline \multirow[b]{3}{*}{ Factor } & \multicolumn{4}{|c|}{ Overall survival } \\
\hline & \multicolumn{2}{|c|}{ Patients } & \multicolumn{2}{|c|}{ Multivariate analysis } \\
\hline & No. & $\%$ & HR $(95 \% \mathrm{CI})$ & P-value \\
\hline All validation set patients & 130 & 100 & & \\
\hline Differentiation & & & & 0.264 \\
\hline Well + moderately & 65 & 50.0 & 1.00 & \\
\hline Poorly & 65 & 50.0 & $1.18(0.88$ to 1.60$)$ & \\
\hline Lauren classification & & & & 0.047 \\
\hline Intestinal & 61 & 46.9 & 1.00 & \\
\hline Diffuse & 69 & 53.1 & 1.73 (1.46 to 3.19$)$ & \\
\hline TNM stage & & & & $<0.001$ \\
\hline $\mathrm{I}+\mathrm{II}$ & 45 & 34.6 & 1.00 & \\
\hline III & 85 & 65.4 & $8.28(3.16$ to 21.70$)$ & \\
\hline TRIM29 expression & & & & 0.003 \\
\hline Low & 61 & 46.9 & 1.00 & \\
\hline High & 69 & 53.1 & $3.32(1.50$ to 7.41$)$ & \\
\hline
\end{tabular}

CI, confidence interval; HR, hazard ratio; TNM, tumor-node-metastasis; TRIM29, tripartite motif containing 29.

HR, 2.81; 95\% CI, 1.56-5.09; $\mathrm{P}=0.001$; TNM stage: HR, 4.93; 95\% CI, 2.32-10.49; P<0.001; TRIM29 expression: HR, 5.68; 95\% CI, 2.58-12.51; $\mathrm{P}<0.001)$ and validation sets (Lauren classification: HR, 1.73; 95\% CI, 1.46-3.19; P=0.047; TNM stage: HR, 8.28; 95\% CI, 3.16-21.70; $\mathrm{P}<0.001$; TRIM29 expression: HR, 3.32; 95\% CI, 1.50-7.41; $\mathrm{P}=0.003$; Table III).

To develop a more sensitive predictive method for patients with gastric cancer, a novel prognostic model combining TNM stage and TRIM29 expression was constructed. ROC analysis was performed to compare its prognostic value against TNM stage or TRIM29 expression alone. The prognostic model that included TNM stage and TRIM29 expression [training set, area under the curve $(\mathrm{AUC})=0.797$; validation set, $\mathrm{AUC}=0.849$ ] was associated with increased prognostic value compared with those that included TNM stage (training set, $\mathrm{AUC}=0.708 ; \mathrm{P}=0.0100$; validation set, $\mathrm{AUC}=0.763 ; \mathrm{P}=0.0001$ ) 
A

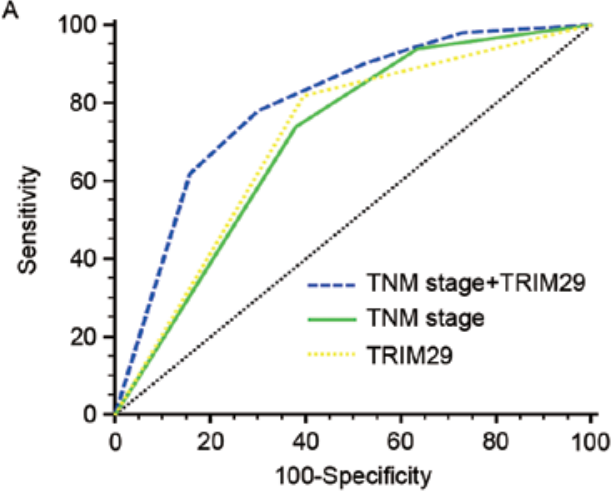

$\begin{array}{lccc} & \text { AUC } & 95 \% \mathrm{Cl} & \text { P } \\ \text { TNM stage + TRIM29 } & 0.797 & 0.711 \text { to } 0.867 & \\ \text { TNM stage } & 0.708 & 0.615 \text { to } 0.790 & 0.0100 \\ \text { TRIM29 } & 0.712 & 0.619 \text { to } 0.793 & 0.0045\end{array}$

B
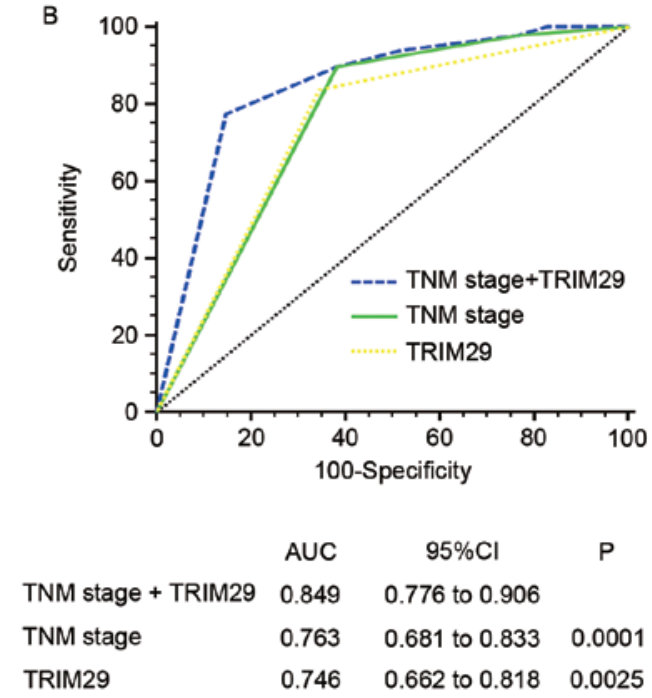

Figure 3. ROC analysis for predicting overall survival in patients with gastric cancer. ROC analysis of the prognostic sensitivity and specificity for overall survival of the TNM stage/TRIM29 expression, TNM stage, and TRIM29 expression models in (A) the training ( $\mathrm{n}=113)$ and $(\mathrm{B})$ validation sets ( $\mathrm{n}=130)$. ROC, receiver operating characteristic; TNM, tumor-node-metastasis; TRIM29, tripartite motif containing 29; AUC, area under the curve; CI, confidence interval; $\mathrm{n}$, number of patients.

or TRIM29 expression (training set, $\mathrm{AUC}=0.712 ; \mathrm{P}=0.0045$; validation set, $\mathrm{AUC}=0.746 ; \mathrm{P}=0.0025)$ alone for the training and validation sets (Fig. 3).

\section{Discussion}

Although the incidence of gastric cancer has decreased during previous decades in numerous industrialized countries, the incidence in China remains one of the highest globally, and the disease has already reached an advanced stage in the majority of patients by the time of diagnosis $(1-3,5)$. The TNM staging system remains an important factor for predicting the prognosis of patients with gastric cancer (4). However, patients with gastric cancer with the same TNM stage may receive different prognoses, partly due to the heterogeneity of the disease at the molecular level (6,7). Identifying specific and sensitive markers to supplement the TNM stage is required to treat patients with gastric cancer more precisely. The present study demonstrated that TRIM29 expression in gastric cancer tissues represents a promising, independent predictor for the survival of patients with gastric cancer. High TRIM29 expression was associated with a decreased overall survival time following surgical resection. Furthermore, incorporating the TRIM29 expression level into the TNM staging system increased the prognostic value of the latter. The results of the present study suggested that TRIM29 expression may possess discriminatory power as a supplementary risk factor in patients with gastric cancer, and facilitate an increase in classification accuracy under the TNM staging system. However, the function of TRIM29 in the prognosis of patients with gastric cancer remains to be fully understood and requires further study.

A previous study identified that TRIM29 was associated with increased aggressiveness in multiple types of cancer (10). TRIM29 antagonizes multiple processes in a number of different cancer cells by regulating certain genes and influencing their functions in numerous cellular signaling pathways (11-16). Another study reported that TRIM29 expression increased as normal pancreatic ductal epithelia progressed to infiltrating cancer, which suggested that upregulating TRIM29 promoted the development of invasive pancreatic cancer (18). However, the underexpression of TRIM29 in breast and prostate cancer has also been reported using serial analysis of gene expression and DNA microarray analysis $(19,20)$. TRIM29 may induce a malignant phenotype to revert to a non-malignant phenotype in osteosarcoma and breast cancer cell lines (21). Zhou et al (22) indicated that upregulating TRIM29 expression promoted proliferation and metastasis in nasopharyngeal carcinoma via the phosphatase and tensin homolog/protein kinase $\mathrm{B} /$ mechanistic target of rapamycin signaling pathway. Kosaka et al (17) reported that TRIM29 expression was positively associated with a poorer histological grade, increased tumor size and invasion, and lymph node metastasis in gastric cancer. In addition, Qiu et al (23) demonstrated that TRIM29 functioned as an oncogene in gastric cancer and was regulated by microRNA-185. However, the association between TRIM29 expression and the prognosis of patients with gastric cancer has not yet been established. The results of the present study revealed that TRIM29 expression was significantly associated with tumor cell differentiation, tumor stage, lymph node metastasis, and TNM stage. Furthermore, an increased percentage of patients with high TRIM29 expression was associated with cancer progression from TNM stage I to III in the training and validation sets. However, elucidating the mechanism underlying the dysregulation of TRIM29 expression in gastric cancer tissues requires further study.

In the present study, all samples were collected from patients at the Cancer Hospital of Henan Province and all surgeries were performed according to the guidelines of the International Gastric Cancer Association and the Japanese Gastric Cancer Association. Immediately following resection of the gastric cancer specimens from the patients, the fresh cancer tissues were frozen and stored in liquid nitrogen until analysis. Prior to RT-qPCR analysis, the specimens were evaluated independently by two gastroenterology pathologists 
blind to clinicopathological patient data. In addition, the experiment was repeated three times with each gastric cancer tissue. However, the present study is limited in certain respects. To begin, the present study is retrospective, and selection bias may not be entirely eliminated. Secondly, data on disease-free survival were not included in the present study and should be collected in subsequent studies. Finally, few patients were enrolled in the present study, confirming the results of which requires large, randomized controlled trials.

To conclude, the results of the present study revealed that TRIM29 expression was associated with unfavorable prognosis and may be adopted as a novel prognostic indicator in patients with resectable gastric cancer. Furthermore, incorporating TRIM29 expression level into the TNM staging system increased the prognostic value of the latter for patients with resectable gastric cancer.

\section{Acknowledgements}

Not applicable.

\section{Funding}

The present study was supported by the Basic and Advanced Technology Research Project of Science and Technology Department of Henan Province (grant no. 142300410276).

\section{Availability of data and materials}

All data generated or analyzed during this study are included in this published article.

\section{Authors' contributions}

$\mathrm{CW}$ and $\mathrm{YZ}$ acquired, analyzed and interpreted the data, performed statistical analysis and drafted the manuscript. BC and WY offered technical and material support. JH designed the study, analyzed and interpreted the data, drafted the manuscript, obtained funding and supervised the study.

\section{Ethics approval and consent to participate}

The present study was reviewed and approved by the Clinical Research Ethics Committee of the Cancer Hospital of Henan Province. All patients provided written informed consent to participate.

\section{Consent for publication}

All patients provided written informed consent.

\section{Competing interests}

The authors declare that they have no competing interests.

\section{References}

1. Chen W, Zheng R, Baade PD, Zhang S, Zeng H, Bray F, Jemal A, Yu XQ and He J: Cancer statistics in China, 2015. CA Cancer J Clin 66: 115-132, 2016
2. Yang L: Incidence and mortality of gastric cancer in China. World J Gastroenterol 12: 17-20, 2006.

3. Chen WQ, Zheng RS, Zhang SW, Zeng HM and Zou XN: The incidences and mortalities of major cancers in China, 2010. Chin J Cancer 33: 402-405, 2014.

4. Washington K: 7th edition of the AJCC cancer staging manual: Stomach. Ann Surg Oncol 17: 3077-3079, 2010.

5. Thrumurthy SG, Chaudry MA, Hochhauser D and Mughal M: The diagnosis and management of gastric cancer. BMJ 347: f6367, 2013.

6. Stadtländer CT and Waterbor JW: Molecular epidemiology, pathogenesis and prevention of gastric cancer. Carcinogenesis 20: 2195-2208, 1999.

7. Figueiredo C, Garcia-Gonzalez MA and Machado JC: Molecular pathogenesis of gastric cancer. Helicobacter 18 (Suppl 1): S28-S33, 2013.

8. Kapp LN, Painter RB, Yu LC, van Loon N, Richard CW III, James MR, Cox DR and Murnane JP: Cloning of a candidate gene for ataxia-telangiectasia group D. Am J Hum Genet 51: 45-54, 1992.

9. Reymond A, Meroni G, Fantozzi A, Merla G, Cairo S, Luzi L, Riganelli D, Zanaria E, Messali S, Cainarca S, et al: The tripartite motif family identifies cell compartments. EMBO J 20: 2140-2151, 2001.

10. Hatakeyama S: Early evidence for the role of TRIM29 in multiple cancer models. Expert Opin Ther Targets 20: 767-770, 2016.

11. Livak KJ and Schmittgen TD: Analysis of relative gene expression data using real-time quantitative PCR and the 2(-Delta Delta C(T)) method. Methods 25: 402-428, 2001.

12. Kanno Y, Watanabe M, Kimura T, Nonomura K, Tanaka S and Hatakeyama S: TRIM29 as a novel prostate basal cell marker for diagnosis of prostate cancer. Acta Histochem 116: 708-712, 2014.

13. Sun H, Dai X and Han B: TRIM29 as a novel biomarker in pancreatic adenocarcinoma. Dis Markers 2014: 317817, 2014.

14. Zhou ZY, Yang GY, Zhou J and Yu MH: Significance of TRIM29 and $\beta$-catenin expression in non-small-cell lung cancer. J Chin Med Assoc 75: 269-274, 2012.

15. Fristrup N, Birkenkamp-Demtröder K, Reinert $T$, Sanchez-Carbayo M, Segersten U, Malmström PU, Palou J, Alvarez-Múgica M, Pan CC, Ulhøi BP, et al: Multicenter validation of cyclin D1, MCM7, TRIM29, and UBE2C as prognostic protein markers in non-muscle-invasive bladder cancer. Am J Pathol 182: 339-349, 2013.

16. Lai W, Zheng X, Huang Q, Wu X and Yang M: Down-regulating ATDC inhibits the proliferation of esophageal carcinoma cells. Eur Rev Med Pharmacol Sci 18: 3511-3516, 2014.

17. Kosaka Y, Inoue H, Ohmachi T, Yokoe T, Matsumoto T, Mimori K, Tanaka F, Watanabe M and Mori M: Tripartite motif-containing 29 (TRIM29) is a novel marker for lymph node metastasis in gastric cancer. Ann Surg Oncol 14: 2543-2549, 2007.

18. Wang L, Heidt DG, Lee CJ, Yang H, Logsdon CD, Zhang L, Fearon ER, Ljungman M and Simeone DM: Oncogenic function of ATDC in pancreatic cancer through Wnt pathway activation and beta-catenin stabilization. Cancer Cell 15: 207-219, 2009.

19. Ernst T, Hergenhahn M, Kenzelmann M, Cohen CD, Bonrouhi M, Weninger A, Klären R, Gröne EF, Wiesel M, Güdemann C, et al: Decrease and gain of gene expression are equally discriminatory markers for prostate carcinoma: A gene expression analysis on total and microdissected prostate tissue. Am J Pathol 160: 2169-2180, 2002

20. Nacht M, Ferguson AT, Zhang W, Petroziello JM, Cook BP, Gao YH, Maguire S, Riley D, Coppola G, Landes GM, et al: Combining serial analysis of gene expression and array technologies to identify genes differentially expressed in breast cancer. Cancer Res 59: 5464-5470, 1999.

21. Hosoi Y, Kapp LN, Murnane JP, Matsumoto Y, Enomoto A, Ono T and Miyagawa K: Suppression of anchorage-independent grow th by expression of the ataxia-telangiectasia group D complementing gene, ATDC. Biochem Biophys Res Commun 348: 728-734, 2006.

22. Zhou XM, Sun R, Luo DH, Sun J, Zhang MY, Wang MH, Yang Y, Wang HY and Mai SJ: Upregulated TRIM29 promotes proliferation and metastasis of nasopharyngeal carcinoma via PTEN/AKT/mTOR signal pathway. Oncotarget 7: 13634-13650, 2016.

23. Qiu F, Xiong JP, Deng J and Xiang XJ: TRIM29 functions as an oncogene in gastric cancer and is regulated by miR-185. Int $\mathrm{J}$ Clin Exp Pathol 8: 5053-5061, 2015. 